\title{
Partonic description of a supersymmetric $p$-brane
}

\author{
KANGHOON LEE ${ }^{\sharp}$ AND JEONG-HYUCK PARK ${ }^{\dagger}$ \\ \#Department of Physics, Yonsei University, Shinchon-dong, Seodaemun-gu, Seoul 120-749, Korea \\ lkh@phya.yonsei.ac.kr \\ ${ }^{\dagger}$ Department of Physics, Sogang University, Shinsu-dong, Mapo-gu, Seoul 121-742, Korea \\ parkesogang.ac.kr
}

\begin{abstract}
We consider supersymmetric extensions of a recently proposed partonic description of a bosonic $p$ brane which reformulates the Nambu-Goto action as an interacting multi-particle action with FilippovLie algebra gauge symmetry. We construct a worldline supersymmetric action by postulating, among others, a $p$-form fermion. Demanding a local worldline supersymmetry rather than the full worldvolume supersymmetry, we circumvent a known no-go theorem against the construction of a RamondNeveu-Schwarz supersymmetric action for a $p$-brane of $p>1$. We also derive a spacetime supersymmetric Green-Schwarz extension from the preexisting kappa-symmetric action.
\end{abstract}

PACS: 11.25.-w, 11.30.Pb

Keywords: parton, $p$-brane, supersymmetry 


\section{Contents}

1 Introduction

2 More on the bosonic action (1.1)

3 Worldline supersymmetry

3.1 Action with foliation preserving local supersymmetry . . . . . . . . . . . 7

3.2 Action with global worldline supersymmetry $\ldots \ldots \ldots \ldots$

4 Spacetime supersymmetry 10

4.1 Action with kappa-symmetry . . . . . . . . . . . . . . . . . . 10

4.2 Action with global spacetime supersymmetry . . . . . . . . . . . . 11

\begin{tabular}{ll|l|} 
& Discussion & 12 \\
\hline
\end{tabular}

\begin{tabular}{ll|} 
A Useful relations & 14 \\
\hline
\end{tabular}

\section{Introduction}

Supersymmetry in string theory is two fold: one on the string worldsheet by the Ramond-Neveu-Schwarz formalism and the other one on the spacetime by the Green-Schwarz formalism. It is known that these two approaches are equivalent, at least for ten-dimensional Minkowskian spacetime. One may attempt to extend the two formalisms to a $p$-brane with $p>1$ i.e. an extended object over $p$-spatial dimensions. Indeed, the extension of the Green-Schwarz covariant superstring action to a $p$-brane is possible for $p \leq 5[1]$. The resulting action is invariant under not only the spacetime supersymmetry but also a fermionic gauge symmetry called kappa-symmetry, such that the on-shell Bose and Fermi degrees of freedom are equal. On the other hand, for a $p$-brane with $p>1$, the Ramond-Neveu-Schwarz extension to the corresponding NambuGoto action reformulated by an auxiliary worldvolume metric [2-4] is known impossible: in Ref. [5] it was shown that the worldvolume supersymmetric extension requires the existence of the Einstein-Hilbert term for the worldvolume metric such that the metric is no longer auxiliary and the connection to the Nambu-Goto action is lost.

\footnotetext{
${ }^{1}$ This action is often dubbed "Polyakov" action.
} 
Recently, a partonic description of a bosonic $p$-brane was proposed in Ref. [6]. With an embedding of $(p+1)$-dimensional worldvolume coordinates into $D$-dimensional target spacetime, $X^{M}\left(\tau, \sigma^{i}\right)$ where and henceforth $i=1, \cdots, p$ and $M=0,1, \cdots, D-1$, the proposed action assumes the form:

$$
S_{\text {bosonic }}=\int \mathrm{d} \tau \operatorname{Tr}\left(\frac{1}{2} D_{\tau} X^{M} D_{\tau} X_{M}-\frac{1}{2 p !}\left\{X^{M_{1}}, X^{M_{2}}, \cdots, X^{M_{p}}\right\}_{\text {N.в. }}\left\{X_{M_{1}}, X_{M_{2}}, \cdots, X_{M_{p}}\right\}_{\text {N.в. }}\right) .
$$

The action contains two kinds of auxiliary fields: the inverse of an einbein $\varphi$ and a gauge connection $A_{\tau}^{i}$. The former defines the trace inside the action,

$$
\operatorname{Tr}(\cdot):=\int \mathrm{d}^{p} \sigma(\varphi \cdot)
$$

and the Nambu bracket 2 [7],

$$
\left\{X^{M_{1}}, X^{M_{2}}, \cdots, X^{M_{p}}\right\}_{\text {N.B. }}:=\varphi^{-1} \epsilon^{i_{1} i_{2} \cdots i_{p}} \partial_{i_{1}} X^{M_{1}} \partial_{i_{2}} X^{M_{2}} \cdots \partial_{i_{p}} X^{M_{p}},
$$

while the latter sets the covariant derivative to be

$$
D_{\tau} X^{M}:=\partial_{\tau} X^{M}-A_{\tau}^{i} \partial_{i} X^{M}
$$

In fact, $\varphi$ and $A_{\tau}^{i}$ may be identified with the "lapse" and "shift" Lagrange multipliers of the Nambu-Goto action in the canonical formalism [8]. Characteristic features of the above action are [6]:

- Integrating out the auxiliary fields, i.e. replacing them by their on-shell values, reduces the action to the standard Nambu-Goto action, as in [2-4] or [9].

- The action is manifestly spacetime Lorentz invariant, despite the similarity to the light-cone gauge fixed actions in [10,11].

- Though not manifest, the action enjoys the full $(p+1)$-dimensional worldvolume diffeomorphism.

- The number of auxiliary component fields is $p+1$, and hence the worldvolume diffeomorphism can fix them completely, such as $\varphi \equiv 1$ and $A_{\tau}^{i} \equiv 0.3$

\footnotetext{
${ }^{2}$ As usual, $\epsilon^{i_{1} i_{2} \cdots i_{p}}$ is the totally anti-symmetric $p$-dimensional tensor density with the normalization $\epsilon^{12 \cdots p}=1$.

${ }^{3}$ c.f. "Polyakov" action where the number of auxiliary component fields is $\frac{1}{2}(p+1)(p+2)$ such that for $p>1$ they can not be gauge fixed completely.
} 
- Partial gauge fixing as $\varphi \equiv 1$ and $\partial_{i} A_{\tau}^{i} \equiv 0$ breaks the worldvolume diffeomorphism to a volume preserving $p$-dimensional diffeomorphism. For a compact $p$-brane this leads to a quantum mechanical system based on Filippov-Lie $p$-algebra 4

- A physical picture behind the reformulation is to describe a single (compact) brane as a collection of interacting multi-particles, and hence the title of this paper: partonic description of a p-brane 5

- One may consider implementing a worldline supersymmetry, rather than the full $(p+1)$-dimensional worldvolume supersymmetry.

In the present paper, we focus on exploring the last property. In the multi-particle description of a (compact) $p$-brane, the temporal worldline direction is singled out from the full $(p+1)$-dimensional worldvolume. Demanding a local supersymmetry along the worldline we may circumvent the aforementioned no-go theorem against the construction of a Ramond-Neveu-Schwarz supersymmetric action for a $p$-brane with $p>16$

Filippov-Lie $p$-algebra appears as a natural generalization of Lie-algebra i.e. two-algebra. While Lie algebra has been extensively studied ever since the inception of Yang-Mills theory, Filippov-Lie $p$-algebra with $p>2$ had not been much explored until 2007 when Bagger-Lambert and Gustavsson employed Filippov-Lie three-algebra with the aim to describe multiple M2-branes [12,13]. In the present paper we shall construct supersymmetric gauge models based on arbitrary Filippov-Lie $p$-algebras.

The organization of the rest of the paper is as follows. In section 2, we review the bosonic action (1.1) with some details, including the full $(p+1)$-dimensional worldvolume diffeomorphism and the Filippov-Lie $p$ algebra regularization. The worldline supersymmetric action is constructed in section 3. We first present a foliation preserving, diffeomorphism invariant and locally supersymmetric Ramond-Neveu-Schwarz action. After gauge fixing we also obtain an action with a global supersymmetry. A crucial ingredient in our worldline supersymmetric extension is to postulate a $p$-form fermion, in addition to a one-form fermion and a gravitino. In section 4 we derive a spacetime supersymmetric Green-Schwarz extension from the known kappa-symmetric action. We write down the proper transformation of the auxiliary fields which will ensure all the symmetries of the spacetime supersymmetric Nambu-Goto action to persist in our refor-

\footnotetext{
${ }^{4}$ Relates works are the Bagger-Lambert-Gustavsson description of multiple M2-branes via Filippov-Lie three-algebra [12 13].

${ }^{5}$ Related works include BFSS $\mathcal{M}$-theory matrix model [15] and Myers' effect [16] etc. See also [11, 17].

${ }^{6}$ For earlier proposals to circumvent the no-go theorem, we refer [18, 19].
} 
mulation. Section 5 contains our discussion and Appendix carries some useful identities.

\section{More on the bosonic action (1.1)}

In this section we review, from Ref. [6], some properties of the bosonic action (1.1) which are relevant to our main results of the supersymmetrization.

With a $p \times p$ matrix defined by

$$
V_{i j}:=\partial_{i} X^{M} \partial_{j} X_{M}
$$

utilizing an identity,

$$
\varphi^{-2} \operatorname{det} V=\frac{1}{p !}\left\{X^{M_{1}}, X^{M_{2}}, \cdots, X^{M_{p}}\right\}_{\text {N.B. }}\left\{X_{M_{1}}, X_{M_{2}}, \cdots, X_{M_{p}}\right\}_{\text {N.B. }},
$$

the bosonic action (1.1) can be rewritten as

$$
S_{\text {bosonic }}=\int \mathrm{d} \tau \mathrm{d}^{p} \sigma\left(\frac{1}{2} \varphi D_{\tau} X^{M} D_{\tau} X_{M}-\frac{1}{2} \varphi^{-1} \operatorname{det} V\right) .
$$

The on-shell values of the auxiliary fields are

$$
A_{\tau}^{i} \equiv \partial_{\tau} X^{M} \partial_{j} X_{M} V^{-1 j i}, \quad \varphi \equiv \sqrt{-\operatorname{det} V /\left(D_{\tau} X^{M} D_{\tau} X_{M}\right)}
$$

Substituting these into the action (2.3), one recovers the Nambu-Goto action [20],

$$
S_{\text {bosonic }} \quad \Longrightarrow \quad S_{\text {N.G. }}=-\int \mathrm{d} \tau \mathrm{d}^{p} \sigma \sqrt{-\operatorname{det}\left(\partial_{\mu} X^{M} \partial_{\nu} X_{M}\right)},
$$

where and henceforth $\mu, \nu$ are the full $(p+1)$-dimensional worldvolume coordinate indices running from zero to $p$. The worldvolume diffeomorphism is realized in rather nontrivial fashion:

$$
\begin{aligned}
& \delta X^{M}=v^{\mu} \partial_{\mu} X^{M}, \\
& \delta \varphi=\partial_{\mu}\left(\varphi v^{\mu}\right)-2 \varphi D_{\tau} v^{\tau}, \\
& \delta A_{\tau}^{i}=D_{\tau} v^{i}-\varphi^{-2} \partial_{j} v^{\tau} V^{-1 j i} \operatorname{det} V+\left(D_{\tau} v^{\tau}+v^{\mu} \partial_{\mu}\right) A_{\tau}^{i},
\end{aligned}
$$

where $v^{\mu}$ is a local parameter having an arbitrary dependence on $\tau$ and $\sigma^{i}$. In general, a symmetry of a given action persists after any reformulation by auxiliary fields: we can always assign transformations to 
the auxiliary fields such that the symmetry is preserved [5]. The above transformation (2.6) is an explicit example of this general statement.

From

$$
\varphi\left(D_{\tau} Y Z+Y D_{\tau} Z\right)=Y Z\left[\partial_{i}\left(\varphi A^{i}\right)-\partial_{\tau} \varphi\right]+\partial_{\tau}(\varphi Y Z)-\partial_{i}\left(\varphi A_{\tau}^{i} Y Z\right)
$$

the vanishing of the following quantity,

$$
\partial_{i}\left(\varphi A_{\tau}^{i}\right)-\partial_{\tau} \varphi=\varphi\left(\partial_{i} A_{\tau}^{i}-D_{\tau} \ln \varphi\right) \equiv 0
$$

is the sufficient and necessary condition of the integration by part for the covariant derivative:

$$
\int \mathrm{d} \tau \operatorname{Tr}\left(D_{\tau} Y Z\right)=-\int \mathrm{d} \tau \operatorname{Tr}\left(Y D_{\tau} Z\right)
$$

with arbitrary $Y$ and $Z$. Under the transformation (2.6),

$$
\begin{aligned}
\delta\left(\partial_{i} A_{\tau}^{i}-D_{\tau} \ln \varphi\right)= & D_{\tau}^{2} v^{\tau}-\frac{1}{(p-1) !}\left\{X^{M_{1}}, \cdots, X^{M_{p-1}},\left\{X_{M_{1}}, \cdots, X_{M_{p-1}}, v^{\tau}\right\}_{\text {N.в. }}\right\}_{\text {N.в. }} \\
& +\left(D_{\tau} v^{\tau}+v^{\mu} \partial_{\mu}\right)\left(\partial_{i} A_{\tau}^{i}-D_{\tau} \ln \varphi\right) .
\end{aligned}
$$

Thus, fixing the gauge (2.8) generically breaks the worldline reparametrization to the global transformation, $v^{\tau}=\alpha \tau+\beta$ with constant parameters $\alpha, \beta$, and reduces the $(p+1)$-dimensional worldvolume diffeomorphism to the $p$-dimensional diffeomorphism on the 'space' part of the worldvolume.

On the other hand, fixing the gauge $\varphi \equiv 1$ and $\partial_{i} A_{\tau}^{i} \equiv 0$, reduces the worldvolume diffeomorphism down to the $p$-dimensional volume preserving diffeomorphism that is subject to the divergence free condition, $\partial_{i} v^{i}=0$. Consequently, the volume preserving gauge symmetry generator as well as the covariant derivative can be represented by the Nambu $p$-bracket: with a functional basis $T^{a}\left(\sigma^{i}\right), a=1,2,3, \cdots$ for the $p$-dimensional manifold which we assume to be compact, we have

$$
\begin{aligned}
& v^{i} \partial_{i}=v_{a_{1} a_{2} \cdots a_{p-1}}\left\{T^{a_{1}}, T^{a_{2}}, \cdots, T^{a_{p-1}}, \quad\right\}_{\text {N.B. }}, \\
& D_{\tau}=\partial_{\tau}-A_{\tau a_{1} a_{2} \cdots a_{p-1}}\left\{T^{a_{1}}, T^{a_{2}}, \cdots, T^{a_{p-1}}, \quad\right\}_{\text {N.B. }} .
\end{aligned}
$$

Note that here $v_{a_{1} a_{2} \cdots a_{p-1}}$ and $A_{\tau a_{1} a_{2} \cdots a_{p-1}}$ depend on $\tau$ only being independent of the $\sigma^{i}$ coordinates. 
As is well known (see e.g. [21]), Nambu $p$-bracket provides an explicit realization of the bracket of the Filippov-Lie $p$-algebra [22], satisfying the totally anti-symmetric property:

$$
\left[X_{1}, \cdots, X_{i}, \cdots, X_{j}, \cdots, X_{p}\right]=-\left[X_{1}, \cdots, X_{j}, \cdots, X_{i}, \cdots, X_{p}\right],
$$

and the Leibniz rule, also known as a fundamental identity:

$$
\left[X_{1}, \cdots, X_{p-1},\left[Y_{1}, \cdots, Y_{p}\right]\right]=\sum_{j=1}^{p}\left[Y_{1}, \cdots,\left[X_{1}, \cdots, X_{p-1}, Y_{j}\right], \cdots, Y_{p}\right] .
$$

In the Nambu bracket realization of the Filippov-Lie algebra, we may employ the structure constant through

$$
\left\{T^{a_{1}}, T^{a_{2}}, \cdots, T^{a_{p}}\right\}_{\text {N.B. }}=f^{a_{1} a_{2} \cdots a_{p}} T^{b} .
$$

The structure constant is then totally anti-symmetric for the upper indices and satisfies from the Leibniz rule (2.13):

$$
f^{a_{1} a_{2} \cdots a_{p}} f^{b_{1} b_{2} \cdots b_{p}} a_{p}=\sum_{j=1}^{p} f^{a_{1} a_{2} \cdots a_{p-1} b_{j}} e f^{b_{1} \cdots b_{j-1} e b_{j+1} \cdots b_{p}} c
$$

Now from (2.11) and 2.14), expanding the dynamical variables by the functional basis, $X^{M}(\tau, \sigma)=$ $X_{a}^{M}(\tau) T^{a}(\sigma)$, the covariant derivative can be rewritten as

$$
D_{\tau} X^{M}=\left(D_{\tau} X^{M}\right)_{a} T^{a}, \quad\left(D_{\tau} X^{M}\right)_{a}=\frac{\mathrm{d}}{\mathrm{d} \tau} X_{a}^{M}-X_{b}^{M} \tilde{A}_{\tau a}^{b}
$$

where we set

$$
\tilde{A}_{\tau a}^{b}:=A_{\tau c_{1} c_{2} \cdots c_{p-1}} f^{c_{1} c_{2} \cdots c_{p-1} b}{ }_{a} .
$$

In this way, the bosonic action (1.1), (2.3) reduces to a genuine quantum mechanical system with gauge symmetry based on an arbitrary Filippov-Lie $p$-algebra. It is worthwhile to note that for $p \geq 3$ the only nontrivial irreducible finite dimensional Filippov-Lie $p$-algebra is, up to signature, so $(p+1)$ [23-26]. With $\tilde{v}^{b}{ }_{a}:=v_{c_{1} c_{2} \cdots c_{p-1}} f^{c_{1} c_{2} \cdots c_{p-1} b}{ }_{a}$, from (2.6) and (2.11), the Filippov-Lie $p$-algebra gauge transformation is given by

$$
\begin{aligned}
& \delta X_{a}^{M}=X_{b}^{M} \tilde{v}_{a}^{b}, \\
& \delta A_{\tau a_{1} a_{2} \cdots a_{p-1}}=\partial_{\tau} v_{a_{1} a_{2} \cdots a_{p-1}}+(-1)^{p}(p-1) A_{\tau c\left[a_{1} a_{2} \cdots a_{p-2}\right.} \tilde{v}_{\left.a_{p-1}\right]}^{c},
\end{aligned}
$$

of which the latter induces, from (2.15),

$$
\delta \tilde{A}_{\tau a}^{b}=\partial_{\tau} \tilde{v}_{a}^{b}-\tilde{v}_{c}^{b} \tilde{A}_{\tau a}^{c}+\tilde{A}_{\tau c}^{b} \tilde{v}_{a}^{c} .
$$

In fact, the case of $p=3$ matches with the Bagger-Lambert-Gustavsson formalism [12, 13]. Other useful relations for the bosonic action are written in Appendix. 


\section{Worldline supersymmetry}

\subsection{Action with foliation preserving local supersymmetry}

The action for the partonic description of a $p$-brane with a local worldline supersymmetry we propose is, with the trace defined in Eq.(1.2):

$$
S_{\text {worldline }}=\int \mathrm{d} \tau \operatorname{Tr}(\hat{\mathcal{L}})
$$

where

$$
\begin{aligned}
\hat{\mathcal{L}}= & \frac{1}{2} D_{\tau} X^{M} D_{\tau} X_{M}-\frac{1}{2 p !}\left\{X^{M_{1}}, X^{M_{2}}, \cdots, X^{M_{p}}\right\}_{\text {N.B. }}\left\{X_{M_{1}}, X_{M_{2}}, \cdots, X_{M_{p}}\right\}_{\text {N.B. }} \\
& +i \frac{1}{2} \psi^{M} D_{\tau} \psi_{M}+i \frac{1}{2 p !} \psi^{M_{1} M_{2} \cdots M_{p}} D_{\tau} \psi_{M_{1} M_{2} \cdots M_{p}} \\
& -i \frac{1}{(p-1) !} \psi^{M_{1} M_{2} \cdots M_{p}}\left\{X_{M_{1}}, X_{M_{2}}, \cdots, X_{M_{p-1}}, \psi_{M_{p}}\right\}_{\text {N.B. }} \\
& +i \chi\left(D_{\tau} X^{M} \psi_{M}+\frac{1}{p !}\left\{X^{M_{1}}, X^{M_{2}}, \cdots, X^{M_{p}}\right\}_{\text {N.B. }} \psi_{M_{1} M_{2} \cdots M_{p}}\right) .
\end{aligned}
$$

In addition to the bosonic fields in 1.1 which are $X^{M}, \varphi, A_{\tau}^{i}$, the above supersymmetric action contains three kinds of fermions: one-form $\psi_{M}, p$-form $\psi_{M_{1} M_{2} \cdots M_{p}}$ and one-dimensional gravitino $\chi$.

The action is invariant under the following foliation preserving diffeomorphism: 7

$$
\begin{aligned}
& \delta X^{M}=v^{\lambda} \partial_{\lambda} X^{M} \\
& \delta \varphi=\partial_{\lambda}\left(v^{\lambda} \varphi\right)-2 \varphi D_{\tau} v^{\tau}, \\
& \delta A_{\tau}^{i}=D_{\tau} v^{i}+\left(D_{\tau} v^{\tau}+v^{\lambda} \partial_{\lambda}\right) A_{\tau}^{i}, \\
& \delta \psi^{M}=v^{\lambda} \partial_{\lambda} \psi^{M}+\frac{1}{2}\left(D_{\tau} v^{\tau}\right) \psi^{M}, \\
& \delta \psi^{M_{1} M_{2} \cdots M_{p}}=v^{\lambda} \partial_{\lambda} \psi^{M_{1} M_{2} \cdots M_{p}}+\frac{1}{2}\left(D_{\tau} v^{\tau}\right) \psi^{M_{1} M_{2} \cdots M_{p}}, \\
& \delta \chi=v^{\lambda} \partial_{\lambda} \chi+\frac{1}{2}\left(D_{\tau} v^{\tau}\right) \chi
\end{aligned}
$$

where

$$
\partial_{i} v^{\tau}=0
$$

\footnotetext{
${ }^{7}$ Under the transformations $(3.3)$, 3.5), the corresponding Lagrangian, $\mathcal{L}_{\text {worldline }}=\varphi \hat{\mathcal{L}}$, transforms to a total derivative.
} 
such that in fact, $D_{\tau} v^{\tau}=\frac{\mathrm{d} v^{\tau}}{\mathrm{d} t}$. Namely while $v^{i}\left(\tau, \sigma^{j}\right)$ is an arbitrary local parameter on the $p$-brane worldvolume, $v^{\tau}(\tau)$ is arbitrary only over the worldline direction and independent of the spatial coordinates $\sigma^{i}$. From the quantum mechanical point of view, the former generates a gauge symmetry, while the latter corresponds to the genuine worldline diffeomorphism. The action is also invariant under a foliation preserving local supersymmetry:

$$
\begin{aligned}
& \delta X^{M}=i \psi^{M} \varepsilon \\
& \delta \varphi=-2 i \varphi \chi \varepsilon \\
& \delta A_{\tau}^{i}=0 \\
& \delta \psi^{M}=D_{\tau} X^{M} \varepsilon \\
& \delta \psi^{M_{1} M_{2} \cdots M_{p}}=\left\{X^{M_{1}}, X^{M_{2}}, \cdots, X^{M_{p}}\right\}_{\text {N.B. }} \varepsilon+i \psi^{M_{1} M_{2} \cdots M_{p}} \chi \varepsilon, \\
& \delta \chi=D_{\tau} \varepsilon+\frac{1}{2}\left(\partial_{i} A_{\tau}^{i}-\varphi^{-1} D_{\tau} \varphi\right) \varepsilon,
\end{aligned}
$$

where $\varepsilon$ is a local fermionic parameter which has arbitrary dependence on the worldline but is independent of the worldvolume spatial coordinates,

$$
\partial_{i} \varepsilon=0
$$

The supersymmetry algebra reads

$$
\delta_{\varepsilon_{1}} \delta_{\varepsilon_{2}}-\delta_{\varepsilon_{2}} \delta_{\varepsilon_{1}}=\delta_{v}
$$

where the right hand side is given by the diffeomorphism parameter,

$$
v^{\tau}=2 i \varepsilon_{1} \varepsilon_{2}, \quad v^{i}=-2 i \varepsilon_{1} \varepsilon_{2} A^{i},
$$

such that the foliation structure is preserved. From the quantum mechanical point of view, the anticommutator of the one-dimensional local supersymmetry amounts to a one-dimensional diffeomorphism plus a gauge symmetry, as usual for supersymmetric gauge theories. Compared to the bosonic action (1.1), the action (3.1) lacks the full $(p+1)$-dimensional worldvolume diffeomorphism, but this is consistent with the no-go theorem against the construction of a worldvolume supersymmetric action for a $p$-brane of $p>1[5]$. 


\subsection{Action with global worldline supersymmetry}

A gauge fixed ( $\varphi \equiv 1$ and $\chi \equiv 0)$ action follows: in terms of the Filippov-Lie $p$-bracket,

$$
\begin{aligned}
\mathcal{L}_{\text {worldline }}^{\prime}= & \frac{1}{2} D_{\tau} X^{M} D_{\tau} X_{M}-\frac{1}{2 p !}\left[X^{M_{1}}, X^{M_{2}}, \cdots, X^{M_{p}}\right]\left[X_{M_{1}}, X_{M_{2}}, \cdots, X_{M_{p}}\right] \\
& +i \frac{1}{2} \psi^{M} D_{\tau} \psi_{M}+i \frac{1}{2 p !} \psi^{M_{1} \cdots M_{p}} D_{\tau} \psi_{M_{1} \cdots M_{p}}-i \frac{1}{(p-1) !} \psi^{M_{1} \cdots M_{p-1} M_{p}}\left[X_{M_{1}}, \cdots, X_{M_{p-1}}, \psi_{M_{p}}\right]
\end{aligned}
$$

The action is clearly invariant under the Filippov-Lie $p$-algebra gauge transformation (2.18), and further enjoys one global worldline supersymmetry: with a constant parameter $\varepsilon_{0}$,

$$
\begin{aligned}
& \delta X^{M}=i \psi^{M} \varepsilon_{0}, \\
& \delta A_{\tau}^{i}=0 \\
& \delta \psi^{M}=D_{\tau} X^{M} \varepsilon_{0}, \\
& \delta \psi^{M_{1} M_{2} \cdots M_{p}}=\left[X^{M_{1}}, X^{M_{2}}, \cdots, X^{M_{p}}\right] \varepsilon_{0} .
\end{aligned}
$$

Especially, when $p=1$ i.e. string, both fermions $\psi^{M}, \psi^{M_{1} M_{2} \cdots M_{p}}$ are on the equal footing carrying only one spacetime index, and there appears an additional SO $(2) R$-symmetry in the action. Consequently the supersymmetry is doubled and the above action reduces, after putting $A_{\tau}^{i} \equiv 0$, to the well-known conformal gauge fixed Ramond-Neveu-Schwarz superstring action [14]. The case of $p=2$ is similar to the BFSS $\mathcal{M}$-theory matrix model [15], but different points in our action are the types of fermions, the worldline supersymmetry and the full target spacetime Lorentz invariance. 


\section{Spacetime supersymmetry}

\subsection{Action with kappa-symmetry}

The spacetime supersymmetric Green-Schwarz covariant $p$-brane Lagrangian reads [1]

$$
-\sqrt{-\operatorname{det}\left(\Pi_{\mu}^{M} \Pi_{\nu M}\right)}+\mathcal{L}_{\text {Wess-Zumino }},
$$

where $\Pi_{\mu}^{M}=\partial_{\mu} X^{M}-i \bar{\theta} \Gamma^{M} \partial_{\mu} \theta$ and $\mathcal{L}_{\text {Wess-Zumino }}$ corresponds to the Wess-Zumino term necessary for the kappa-symmetry. The super $p$-brane action exists if and only if the Bose and Fermi degrees of freedom match, such that the possible values of $p$ and the spacetime dimension $D$ are

$$
\begin{array}{ll}
p=1: & D=3,4,6,10 \\
p=2: & D=4,5,7,11 \\
p=3: & D=6,8 \\
p=4: & D=9 \\
p=5: & D=10 .
\end{array}
$$

Our partonic reformulation of the spacetime supersymmetric Green-Schwarz $p$-brane Lagrangian is then:

$$
\mathcal{L}_{\text {spacetime }}=\mathcal{L}\left(\varphi, A_{\tau}^{i}, \Pi_{\mu}^{M}\right)+\mathcal{L}_{\text {Wess-Zumino }},
$$

where, with $\mathcal{V}_{i j}:=\Pi_{i}^{M} \Pi_{j M}$,

$$
\mathcal{L}\left(\varphi, A_{\tau}^{i}, \Pi_{\mu}^{M}\right)=\frac{1}{2} \varphi\left(\Pi_{\tau}^{M}-A_{\tau}^{i} \Pi_{i}^{M}\right)\left(\Pi_{\tau M}-A_{\tau}^{j} \Pi_{j M}\right)-\frac{1}{2} \varphi^{-1} \operatorname{det} \mathcal{V} .
$$

In particular, in a similar fashion to (2.2), we may write [27]

$$
\begin{aligned}
& \varphi^{-2} \operatorname{det} \mathcal{V}=\frac{1}{p !}\left\langle\Pi^{M_{1}}, \Pi^{M_{2}}, \cdots, X^{M_{p}}\right\rangle\left\langle\Pi_{M_{1}}, \Pi_{M_{2}}, \cdots, \Pi_{M_{p}}\right\rangle, \\
& \left\langle\Pi^{M_{1}}, \Pi^{M_{2}}, \cdots, X^{M_{p}}\right\rangle:=\varphi^{-1} \epsilon^{i_{1} i_{2} \cdots i_{p}} \Pi_{i_{1}}^{M_{1}} \Pi_{i_{2}}^{M_{2}} \cdots \Pi_{i_{p}}^{M_{p}} .
\end{aligned}
$$

The auxiliary fields assume the following on-shell values,

$$
\begin{aligned}
& A_{\tau}^{i} \quad \Longrightarrow \quad \hat{A}_{\tau}^{i}:=\Pi_{\tau}^{M} \Pi_{j M} \mathcal{V}^{-1 j i} \\
& \varphi \quad \Longrightarrow \quad \hat{\varphi}:=\sqrt{-\frac{\operatorname{det} \mathcal{V}}{\left(\Pi_{\tau}^{M}-\hat{A}_{\tau}^{i} \Pi_{i}^{M}\right)\left(\Pi_{\tau M}-\hat{A}_{\tau}^{j} \Pi_{j M}\right)}} .
\end{aligned}
$$


Integrating them out reduces $\mathcal{L}\left(\varphi, A_{\tau}^{i}, \Pi_{\mu}^{M}\right)$ to the supersymmetric Nambu-Goto term in (4.1),

$$
\mathcal{L}\left(\varphi, A_{\tau}^{i}, \Pi_{\mu}^{M}\right) \quad \Longrightarrow \quad \mathcal{L}\left(\hat{\varphi}, \hat{A}_{\tau}^{i}, \Pi_{\mu}^{M}\right)=-\sqrt{-\operatorname{det}\left(\Pi_{\mu}^{M} \Pi_{\nu M}\right)}
$$

Furthermore, along with an arbitrary transformation $\delta \Pi_{\mu}^{M}$, if we let the auxiliary fields transform as

$$
\begin{aligned}
& \delta A_{\tau}^{i}=\delta \hat{A}_{\tau}^{i}+\frac{1}{2}\left(\hat{A}_{\tau}^{i}-A_{\tau}^{i}\right) \delta \ln \varphi+\left(\hat{A}_{\tau}^{k}-A_{\tau}^{k}\right) \Pi_{k}^{M} \delta \Pi_{j M} \mathcal{V}^{-1 j i}, \\
& \delta \varphi=\frac{2 \varphi^{2}}{(\hat{\varphi}+\varphi) \hat{\varphi}} \delta \hat{\varphi}+\frac{(\hat{\varphi}-\varphi) \varphi}{\hat{\varphi}+\varphi} \delta \ln \operatorname{det} \mathcal{V}
\end{aligned}
$$

the variation of $\mathcal{L}\left(\varphi, A_{\tau}^{i}, \Pi_{\mu}^{M}\right)$ becomes independent of the auxiliary fields and, moreover, coincides with that of the spacetime supersymmetric Nambu-Goto term:

$$
\delta \mathcal{L}\left(\varphi, A_{\tau}^{i}, \Pi_{\mu}^{M}\right)=\delta \mathcal{L}\left(\hat{\varphi}, \hat{A}_{\tau}^{i}, \Pi_{\mu}^{M}\right)=-\delta \sqrt{-\operatorname{det}\left(\Pi_{\mu}^{M} \Pi_{\nu M}\right)} .
$$

Therefore, all the symmetries of the Green-Schwarz super $p$-brane Lagrangian (4.1) survive in our partonic reformulation (4.3), which include the spacetime supersymmetry, the spacetime Lorenz symmetry, the kappa-symmetry and the worldvolume diffeomorphism 8

\subsection{Action with global spacetime supersymmetry}

The light-cone gauge fixed actions are ready to be read-off from an earlier work by Bergshoeff, Sezgin, Tanii and Townsend [11]. In its appendix the authors listed light-cone gauge fixed supersymmetric actions for various $p$-branes in diverse spacetime dimensions. Utilizing the identity (2.2), in terms of Filippov-Lie algebra $p$-bracket, their light-cone gauge fixed spacetime supersymmetric $p$-brane actions can be rewritten in a compact form:

$$
\mathcal{L}_{\text {spacetime }}^{\prime}=\frac{1}{2}\left(D_{t} X^{I}\right)^{2}-\frac{1}{2 p !}\left[X^{I_{1}}, X^{I_{2}}, \cdot \cdot, X^{I_{p}}\right]^{2}+i \frac{1}{2} \bar{\theta} D_{t} \theta+\frac{1}{2(p-1) !} \bar{\theta} \Gamma^{I_{1} I_{2} \cdots I_{p-1}}\left[X_{I_{1}}, \cdots, X_{I_{p-1}}, \theta\right] .
$$

Here the spacetime index $I$ runs from one to $D-2$ with the possible values of $p$ and $D$ in (4.2). For details of the supersymmetry transformation we refer to Ref. [11], 9

\footnotetext{
${ }^{8}$ See [27, 28] for similar works, and recall the general phenomenon that no symmetry is lost under an arbitrary reformulation of a given action by auxiliary fields [5].

${ }^{9}$ See also Ref. [29] for a zero-dimensional analogy.
} 


\section{Discussion}

In this paper we have constructed supersymmetric extensions of a bosonic $p$-brane action which reformulates the Nambu-Goto action as an interacting multi-particle action with Filippov-Lie $p$-algebra gauge symmetry. We obtained a worldline supersymmetric action by postulating, among others, a $p$-form fermion. We also derived a spacetime supersymmetric Green-Schwarz extension from the preexisting kappa-symmetric action.

Compared to the ordinary Lie algebra, one limited feature of Filippov-Lie $p$-algebra for $p \geq 3$ is that, finite dimensional irreducible Filippov-Lie algebra is essentially unique, i.e. $\mathbf{s o}(p+1)[23-26]$. Consequently there is no arbitrary tunable parameter as for the number of finite degrees of freedom. One should deal with infinite dimensional Filippov-Lie $p$-algebras or $\mathbf{s o}(p+1)$. In the latter case the $p$-brane corresponds to a fuzzy $p$-sphere.

For $p=2$, the situation is different. We may safely adopt matrices of arbitrary size. With the usual matrix commutator, our actions read from (3.9),

$$
\mathcal{L}_{\text {worldline }}=\frac{1}{2} D_{\tau} X^{M} D_{\tau} X_{M}-\frac{1}{4}\left[X^{M}, X^{N}\right]^{2}+i \frac{1}{2} \psi^{M} D_{\tau} \psi_{M}+i \frac{1}{4} \psi^{M N} D_{\tau} \psi_{M N}-i \psi^{M N}\left[X_{M}, \psi_{N}\right]
$$

and from (4.10),

$$
\mathcal{L}_{\text {spacetime }}=\frac{1}{2} D_{t} X^{I} D_{t} X_{I}-\frac{1}{4}\left[X^{M}, X^{N}\right]^{2}+i \frac{1}{2} \bar{\theta} D_{t} \theta+\frac{1}{2} \bar{\theta} \Gamma^{I}\left[X_{I}, \theta\right] .
$$

Of course, the latter corresponds to the well-known BFSS $\mathcal{M}$-theory matrix model [15] where the fermion is a target spacetime spinor. In analogy with the equivalence between the RNS and the GS superstring actions, it is crucial to check the connection between the above two actions for M2-brane.

In the case of $p=0$, with vanishing Nambu bracket, our worldline supersymmetric action (3.1) reduces to the well-known action for a massless supersymmetric particle (see e.g. [30]). Our action then corresponds to non-Abelian or Filippov-Lie algebra generalization of it, where a 'mass' term appears as the square of Nambu bracket. We recall the known difficulty that a massive point-particle does not allow a worldline supersymmetric extension, as there is no supersymmetric counter part to its mass term,

$$
S=\frac{1}{2} \int \mathrm{d} \tau\left(e \partial_{\tau} X^{M} \partial_{\tau} X_{M}-e^{-1} m^{2}\right)
$$

While a compact $p$-brane should look like a point-particle at far distance, our result delivers a novel way of introducing a supersymmetric mass term: the Filippov-Lie algebra based interaction of the partons of the 
compact $p$-brane gives rise to the mass term. Namely, mass originates from the internal interaction, like a proton made of light quarks. Further, we expect that the constraint form the equation of motion for $\chi 10$

$$
\psi^{M} D_{\tau} X_{M}+\frac{1}{p !} \psi^{M_{1} M_{2} \cdots M_{p}}\left\{X_{M_{1}}, X_{M_{2}}, \cdots, X_{M_{p}}\right\}_{\mathrm{N} . \mathrm{B} .}=0,
$$

also leads, after quantization, to a certain massive Dirac equation. We leave the quantization of the supersymmetric $p$-brane in our formulation for future work. In the present paper we focused on the partonic description of a super $p$-brane. Since the aforementioned no-go theorem prohibits the construction of a worldvolume supersymmetric $p$-brane action for $p>1$, the other alternative possibility worth trying is the supersymmetric extensions of the 'multi-string description of a $p$-brane,' starting from the bosonic action [6],

$$
\begin{aligned}
& S_{\text {string }}=\int \mathrm{d}^{2} \tau \operatorname{Tr}\left(\sqrt{-h} \mathcal{L}_{\text {string }}\right), \quad \operatorname{Tr}:=\int \mathrm{d}^{p-1} \sigma, \\
& \mathcal{L}_{\text {string }}=-e^{-\phi} h^{a b} D_{a} X^{M} D_{b} X_{M}-\frac{1}{4} e^{\phi} \operatorname{det} V+e^{-\phi},
\end{aligned}
$$

where $a, b=0,1$ are the two-dimensional worldsheet indices [34]. The resulting action may correspond to a RNS version of the well-known matrix string action [35].

\section{Acknowledgements}

We thank Seungjoon Hyun and Corneliu Sochichiu for helpful comments. The work is supported by the National Research Foundation of Korea(NRF) grant funded by the Korea government(MEST) through the Center for Quantum Spacetime(CQUeST) of Sogang University with grant number 2005-0049409, and by the National Research Foundation of Korea(NRF) grant funded by the Korea government(MEST) (No. 2009-0083765).

\footnotetext{
${ }^{10}$ c.f. [31, 32] and some BPS equations in the Bagger-Lambert-Gustavsson theory [33].
} 


\section{A Useful relations}

Here we write some useful identities:

$$
\begin{gathered}
\left\{X^{\left[M_{1}\right.}, \cdots, X^{M_{p}}\right\}_{\text {N.B. }} \partial_{i} X^{\left.M_{p+1}\right]}=0 \quad \text { for arbitrary } i \\
\left\{X_{\left[M_{1}\right.}, \cdots, X_{M_{p-1}}, v^{i}\right\}_{\text {N.B. }} \partial_{i} X_{\left.M_{p}\right]}=\frac{1}{p} \partial_{i} v^{i}\left\{X_{M_{1}}, \cdots, X_{M_{p}}\right\}_{\text {N.B. }}, \\
\varphi^{-2} \operatorname{det} V V^{-1 i j} \partial_{i} Y \partial_{j} Z=\frac{1}{(p-1) !}\left\{X^{M_{1}}, X^{M_{2}}, \cdots, X^{M_{p-1}}, Y\right\}_{\text {N.B. }}\left\{X_{M_{1}}, X_{M_{2}}, \cdots, X_{M_{p-1}}, Z\right\}_{\text {N.B. }}, \\
\varphi^{-1} \partial_{i}\left(\varphi^{-1} \operatorname{det} V V^{-1 i j} \partial_{j} Y\right)=\frac{1}{(p-1) !}\left\{X^{M_{1}}, X^{M_{2}}, \cdots, X^{M_{p-1}},\left\{X_{M_{1}}, X_{M_{2}}, \cdots, X_{M_{p-1}}, Y\right\}_{\text {N.B. }}\right\}_{\text {N.B. }}, \\
\operatorname{det} V=\frac{1}{p !} \epsilon^{r_{1} r_{2} \cdots r_{p}} \epsilon^{s_{1} s_{2} \cdots s_{p}} V_{r_{1} s_{1}} V_{r_{2} s_{2}} \cdots V_{r_{p} s_{p}}, \\
\frac{\partial^{n} \operatorname{det} V}{\partial V_{i_{1} j_{1}} \partial V_{i_{2} j_{2}} \cdots \partial V_{i_{n} j_{n}}}=\frac{1}{(p-n) !} \epsilon^{i_{1} \cdots i_{n} r_{1} \cdots r_{p-n}} \epsilon^{j_{1} \cdots j_{n} s_{1} \cdots s_{p-n}} V_{r_{1} s_{1}} \cdots V_{r_{p-n} s_{p-n}} .
\end{gathered}
$$

In particular,

$$
\begin{aligned}
& V^{-1 i j} \operatorname{det} V=\frac{1}{(p-1) !} \epsilon^{i r_{1} \cdots r_{p-1}} \epsilon^{j s_{1} \cdots s_{p-1}} V_{r_{1} s_{1}} \cdots V_{r_{p-1} s_{p-1}} \\
& \left(V^{-1 i j} V^{-1 k l}-V^{-1 i k} V^{-1 l j}\right) \operatorname{det} V=\frac{1}{(p-2) !} \epsilon^{i k r_{1} \cdots r_{p-2}} \epsilon^{j l s_{1} \cdots s_{p-2}} V_{r_{1} s_{1}} \cdots V_{r_{p-2} s_{p-2}} .
\end{aligned}
$$

Under the worldvolume diffeomorphism (2.6),

$$
\begin{aligned}
\delta D_{\tau} X^{M}= & \frac{1}{(p-1) !}\left\{X^{N_{1}}, \cdots, X^{N_{p-1}}, v^{\tau}\right\}_{\text {N.в. }}\left\{X_{N_{1}}, \cdots, X_{N_{p-1}}, X^{M}\right\}_{\text {N.B. }} . \\
& +\left(D_{\tau} v^{\tau}+v^{\mu} \partial_{\mu}\right) D_{\tau} X^{M}, \\
\delta\left\{X^{M_{1}}, \cdots, X^{M_{p}}\right\}_{\text {N.B. }}= & \sum_{k=1}^{p} D_{\tau} X^{M_{k}}\left\{X^{M_{1}}, \cdots, X^{M_{k-1}}, v^{\tau}, X^{M_{k+1}}, \cdots, X^{M_{p}}\right\}_{\text {N.B. }} . \\
& +\left(D_{\tau} v^{\tau}+v^{\mu} \partial_{\mu}\right)\left\{X^{M_{1}}, \cdots, X^{M_{p}}\right\}_{\text {N.в. }} .
\end{aligned}
$$




\section{References}

[1] A. Achucarro, J. M. Evans, P. K. Townsend and D. L. Wiltshire, Phys. Lett. B 198 (1987) 441.

[2] S. Deser and B. Zumino, Phys. Lett. B 65 (1976) 369.

[3] L. Brink, P. Di Vecchia and P. S. Howe, Phys. Lett. B 65 (1976) 471.

[4] P. S. Howe and R. W. Tucker, J. Phys. A 10 (1977) L155.

[5] E. Bergshoeff, E. Sezgin and P. K. Townsend, Phys. Lett. B 209 (1988) 451.

[6] J.-H. Park and C. Sochichiu, Eur. Phys. J. C 64 (2009) 161-166 [arXiv:0806.0335 hep-th].

[7] Y. Nambu, Phys. Rev. D 72405 (1973).

[8] M. Henneaux, Phys. Lett. B 120 (1983) 179.

[9] A. Schild, Phys. Rev. D 16 (1977) 1722.

[10] J. Hoppe, MIT PhD thesis 1982 and Elem. Part. Res. J. (Kyoto) 80 (1989) 145;

J. Hoppe, Helv. Phys. Acta 70 (1997) 302 [arXiv:hep-th/9602020].

[11] E. Bergshoeff, E. Sezgin, Y. Tanii and P. K. Townsend, Annals Phys. 199 (1990) 340.

[12] J. Bagger and N. Lambert, Phys. Rev. D 75 (2007) 045020 [arXiv:hep-th/0611108];

J. Bagger and N. Lambert, Phys. Rev. D 77 (2008) 065008 [arXiv:0711.0955 [hep-th]];

J. Bagger and N. Lambert, JHEP 0802 (2008) 105 [arXiv:0712.3738][hep-th]].

[13] A. Gustavsson, Nucl. Phys. B 811, 66 (2009) [arXiv:0709.1260 [hep-th]].

[14] M. B. Green, J. H. Schwarz and E. Witten, "SUPERSTRING THEORY. VOL. 1: INTRODUCTION," Cambridge, Uk: Univ. Pr. (1987).

[15] T. Banks, W. Fischler, S. H. Shenker and L. Susskind, Phys. Rev. D 55 (1997) 5112 arXiv:hep-th/9610043.

[16] R. C. Myers, JHEP 9912 (1999) 022 [arXiv:hep-th/9910053].

[17] H. S. Yang, I. Kim and B. H. Lee, Phys. Rev. D 58 (1998) 085018 [arXiv:hep-th/9806112]. 
[18] U. Lindstrom and M. Rocek, Phys. Lett. B 218 (1989) 207.

[19] C. Castro, Phys. Lett. B 559 (2003) 74 [arXiv:hep-th/0212022].

[20] Y. Nambu, Lectures at the Copenhagen symposium, 1970; T. Goto, Prog. Theor. Phys. 46 (1971) 1560.

[21] L. Takhtajan, Commun. Math. Phys. 160 (1994) 295 [arXiv:hep-th/9301111];

H. Awata, M. Li, D. Minic and T. Yoneya, JHEP 0102 (2001) 013 [arXiv:hep-th/9906248].

[22] V. T. Filippov, “n-Lie algebras,” Sib. Mat. Zh., 26, No 6, 126-140 (1985).

[23] J. M. Figueroa-O'Farrill and G. Papadopoulos, arXiv:math/0211170.

[24] G. Papadopoulos, JHEP 0805 (2008) 054 [arXiv:0804.2662 [hep-th]].

[25] J. P. Gauntlett and J. B. Gutowski, arXiv:0804.3078 [hep-th].

[26] G. Papadopoulos, Class. Quant. Grav. 25 (2008) 142002 [arXiv:0804.3567[hep-th]].

[27] K. Lee and J.-H. Park, JHEP 0904 (2009) 012 [arXiv:0902.2417[hep-th]].

[28] D. Kamani, arXiv:0904.2721v3 [hep-th].

[29] K. Furuuchi and D. Tomino, JHEP 0905 (2009) 070 [arXiv:0902.2041 [hep-th]].

[30] J. Gomis, J. Paris and S. Samuel, Phys. Rept. 259 (1995) 1 [arXiv:hep-th/9412228].

[31] G. Bonelli and M. Zabzine, JHEP 0509 (2005) 015 [arXiv:hep-th/0507051].

[32] G. Bonelli, A. Tanzini and M. Zabzine, Phys. Lett. B 672 (2009) 390 [arXiv:0807.5113 [hep-th]].

[33] I. Jeon, J. Kim, N. Kim, S. W. Kim and J.-H. Park, JHEP 0807 (2008) 056 [arXiv:0805.3236 [hep-th]].

[34] K. Lee and J.-H. Park in preparation.

[35] R. Dijkgraaf, E. P. Verlinde and H. L. Verlinde, Nucl. Phys. B 500 (1997) 43 [arXiv:hep-th/9703030]. 